\title{
A METHOD TO IMPROVE CONSISTENCY OF THE COMPARISON MATRIX BASED ON ITERATION
}

\author{
Kazutomo Nishizawa \\ Department of Mathematical Engineering, \\ College of Industrial Technology, Nihon University \\ 1-2-1 Izumi-cho, Narashino, Chiba 275-8575, Japan \\ k7nisiza@ccu.cit.nihon-u.ac.jp
}

\begin{abstract}
AHP (Analytic Hierarchy Process) is a useful tool for decision-makers. In AHP, it is important to evaluate a consistency of comparisons. In this paper, we propose a method to improve consistency of the comparison matrix. In inconsistent case, various improvement methods were proposed. However, these methods are to correct some part of comparison matrix. For the proposed method, we are intend to adjust the whole element of the comparison matrix based on iteration. We illustrate the usefulness of our method through several examples.
\end{abstract}

\section{Introduction.}

AHP (Analytic Hierarchy Process) [Saaty,1977] [Saaty,1980] is widely used for decision makers in various fields. In AHP, it is important to evaluate a consistency of comparisons. In this paper we propose a method to improve consistency based on itetation. The following symbols are basically used in this paper: The comparison matrix of $n$ alternatives is $\boldsymbol{A}$ and the element of $\boldsymbol{A}$ is $a_{i j}(i=1 \sim n, j=1 \sim n)$. Maximum eigenvalue of $A$ is $\lambda_{m a x}$ and corresponding eigenvector is $W$ and the element of $W$ is $w_{i}(i=1 \sim n)$.

In general, a consistency is measured by $C I$ ( Consistency Index ), as shown below,

$$
C I=\left(\lambda_{\max }-n\right) /(n-1)
$$

and if $C I<0.1$, the comparison matrix is consistent. In a perfect consistent case, the element of comparison matrix is represented by ratio of the alternative's weights, that is any alternatives $i$ and $j$ we have

$$
a_{i j}=w_{i} / w_{j} \text {. }
$$

In actual pairwise comparison, caused by overestimate or underestimate, $a_{i j}$ is include error $e_{i j}$, however, it is not easy to estimate $e_{i j}$. Then eq.(2) becomes

$$
a_{i j}=\frac{w_{i}}{w_{j}} e_{i j}
$$

In inconsistent case, various improvement methods were proposed. For example, one is based on ratio of the alternative's weights[Tone, 1990], the other is based on directed graph of comparison matrix[Nishizawa,1995]. For the former method, firstly, compare $a_{i j}$ with ratio of the obtained weights $w_{i} / w_{j}$ and find the biggest one of differences $\left(a_{i j}-w_{i} / w_{j}\right)$, and next, correct the corresponding element of the comparison matrix. For the latter method, firstly, draw a directed graph of the comparison matrix whose edges are represented by " $i \rightarrow j$ ", if alternative $i$ is more important than alternative $j$. If there are some cycles in the directed graph, these cycles are considered as the cause of inconsistent. Next find the cycles[Nishizawa,1997] and correct the effective elements for eliminating the cycles. 
The above 2 kind methods are to correct some part of comparison matrix, while the proposed method intends to adjust the comparison matrix as the whole. In section 2, our concept and improvement procedure are described. In section 3, we illustrate the usefulness of our method through the examples. Finally in section 4 , we conclude our investigation.

\section{Proposed method}

In this section, we describe the proposed method to improve consistency of the comparison matrix. The concept of our method is based on iteration.

From eq.(2), we can rewrite the perfect consistent comparison matrix $\boldsymbol{A}$ as follows.

$$
\boldsymbol{A}=\left[\begin{array}{cccc}
w_{1} / w_{1} & w_{1} / w_{2} & \cdots & w_{1} / w_{n} \\
w_{2} / w_{1} & w_{2} / w_{2} & \cdots & w_{2} / w_{n} \\
\vdots & \vdots & \vdots & \vdots \\
w_{n} / w_{1} & w_{n} / w_{2} & \cdots & w_{n} / w_{n}
\end{array}\right]
$$

For any $k$, based on eq.(2), we have below equation.

$$
a_{i j}=\left(w_{i} / w_{k}\right) /\left(w_{j} / w_{k}\right)=a_{i k} / a_{j k}=a_{k j} / a_{k i}
$$

Therefore $a_{i j}$ is represented by 2 elements of $\boldsymbol{A}$. So, for each $k=1 \sim n$, we have $a_{i j_{k}}^{\prime}$ from below equation.

$$
a_{i j_{k}}^{\prime}=a_{k j} / a_{k i}
$$

However, caused by the error term, the results of eq.(6) are not coincide. Then by applying geometric mean to $a_{i j_{k}}^{\prime}$, as shown below, we intend to reduce the error.

$$
a_{i j}^{\prime}=\left(\prod_{k=1}^{n} a_{i j_{k}}^{\prime}\right)^{1 / n}
$$

Proposed procedure, mentiond above, is summarized as follows:

(1) For each $k=1 \sim n$, calculate $a_{i j_{k}}^{\prime}$ from eq. (6).

(2) Calculate $a_{i j}^{\prime}$, geometric mean of $a_{i j_{k}}^{\prime}$, from eq.(7).

(3) Rewrite $\boldsymbol{A}$ as $a_{i j}=a_{i j}^{\prime}$ and calculate $\lambda_{\max }$.

(4) Repeat (1) to (3) untill $\lambda_{\max }$ converge $n$.

Moreover, in the next section, we describe our method in detail through the examples.

\section{Example}

In this section, in order to confirm the usefulness of our concept, we apply proposed method to the examples with 5 alternatives $(n=5)$. We artificially add error to each elements of the perfect consistent comparison matrix. Our improvement method is carried out and obtained weights are evaluated by $R S S$ (Residual Sum of Squares).

Firstly we construct the perfect consistent comparison matrix. Suppose the correct weights are $w_{i}=i(i=1 \sim 5)$, as shown below.

$$
W=\left[\begin{array}{lllll}
1 & 2 & 3 & 4 & 5
\end{array}\right]
$$

Normalizing with sum of the elements of $W$ equal to 1 , we have the following weights.

$$
W=\left[\begin{array}{lllll}
.06666 & .13333 & .20000 & .26666 & .33333
\end{array}\right]
$$


Based on eq.(2) and eq.(9), we have the perfect consistent comparison matrix $A_{0}$, as shown below. Of course $\lambda_{\max }=5$ and $C I=0$.

$$
A_{o}=\left[\begin{array}{lllll}
1.0000 & 0.5000 & 0.3333 & 0.2500 & 0.2000 \\
2.0000 & 1.0000 & 0.6666 & 0.5000 & 0.4000 \\
3.0000 & 1.4999 & 1.0000 & 0.7500 & 0.6000 \\
4.0000 & 2.0000 & 1.3333 & 1.0000 & 0.8000 \\
5.0000 & 2.5000 & 1.6666 & 1.2500 & 1.0000
\end{array}\right]
$$

Secondly we artificially add $e_{i j}$ to each elements of $A_{0}$. By using the logarithmic scale, eq.(3) is represented by the following equation.

$$
\log a_{i j}=\log a_{k j}-\log a_{k i}+\log e_{i j}
$$

To construct inconsistent comparison matrix, we chose $\log e_{i j}$ as normal random numbers of $\mathrm{N}\left(0, \sigma^{2}\right)$, where $\sigma$ is the standard deviation.

Finally, we calculate $R S S$ between improved weights and eq.(9), and evaluate the result.

In this section we illustrate our method by two kinds of examples. With respect to $C I$, one is very close to 0.1 , and the other is greater than 0.1 .

\section{Example 1}

By using proposed method, we improve the comparison matrix whose $C I$ is very close to 0.1 .

Firstly, add $\log e_{i j} \in \mathrm{N}\left(0,1^{2}\right)$ to $\boldsymbol{A}_{\mathbf{0}}$, we construct the comparison matrix $\boldsymbol{A}_{\mathbf{1}}$, as shown below.

$$
A_{\mathbf{z}}=\left[\begin{array}{lllll}
1.0000 & 0.3778 & 0.8931 & 0.2694 & 0.2745 \\
2.6463 & 1.0000 & 0.2804 & 0.7188 & 0.5922 \\
1.1196 & 3.5650 & 1.0000 & 0.7383 & 0.5701 \\
3.7115 & 1.3911 & 1.3543 & 1.0000 & 0.8799 \\
3.6428 & 1.6883 & 1.7539 & 1.1364 & 1.0000
\end{array}\right]
$$

From $A_{1}$, we have $\lambda_{\max }=5.396$ and $C I=0.099$, however, there are no cycles in corresponding directed graph.

Next, let us explain the improvement process of $a_{1_{12}}$, the element of $A_{1}$, by using proposed method. From eq.(6), we have below values.

$$
\begin{aligned}
& a_{1_{121}}^{\prime}=a_{1_{12}} / a_{1_{11}}=0.3778 / 1.0000=0.3778 \\
& a_{1_{222}}^{\prime}=a_{1_{22}} / a_{1_{21}}=1.0000 / 2.6463=0.3778 \\
& a_{1_{123}}^{\prime}=a_{1_{32}} / a_{1_{31}}=3.5650 / 1.1196=3.1841 \\
& a_{1_{124}}^{\prime}=a_{1_{42}} / a_{1_{41}}=1.3911 / 3.7115=0.3748 \\
& a_{1_{125}}^{\prime}=a_{1_{52}} / a_{1_{81}}=1.6883 / 3.6428=0.4634
\end{aligned}
$$

From eq.(7), we have $a_{1_{13}}^{\prime}=0.6018$. By similar procedure, by once iteration, we have improved comparison matrix $A_{1}^{\prime}$, as shown below.

$$
\boldsymbol{A}_{\mathbf{1}}^{\prime}=\left[\begin{array}{lllll}
1.0000 & 0.6018 & 0.4308 & 0.3323 & 0.2895 \\
1.6614 & 1.0000 & 0.7159 & 0.5522 & 0.4811 \\
2.3207 & 1.3968 & 1.0000 & 0.7713 & 0.6720 \\
3.0086 & 1.8108 & 1.2963 & 1.0000 & 0.8712 \\
3.4533 & 2.0785 & 1.4880 & 1.1478 & 1: 0000
\end{array}\right]
$$

From $A_{1}^{\prime}$ we have $\lambda_{\max }=5$ and $C I=0$, and there are no new cycles in corresponding directed graph. 
Table 1. Each weight and corresponding RSS of Example 1

\begin{tabular}{ccccccc}
\hline & $w_{1}$ & $w_{2}$ & $w_{3}$ & $w_{4}$ & $w_{5}$ & $R S S\left(\times 10^{-3}\right)$ \\
\hline$A_{0}$ & .06666 & .13333 & .20000 & .26666 & .33333 & \\
$A_{1}$ & .09134 & .14875 & .22309 & .25027 & .28653 & 3.8388 \\
$A_{1}^{\prime}$ & .08738 & .14517 & .20278 & .26289 & .30175 & 1.5887 \\
\hline
\end{tabular}

Each weight of $A_{0}, A_{1}$ and $A_{1}^{\prime}$ and corresponding $R S S$ are shown in Table 1.

As a result, from Table 1 , it is clear that each weight of $A_{1}^{\prime}$ is closer to the correct weight than each weight of $A_{1}$. Of course with respect to $R S S, A_{1}^{\prime}$ is better than $A_{1}$.

\section{Example 2}

Example 2 is more inconsistent case, whose $C I$, is far greater than 0.1 . The improving procedure is similar as Example 1.

Firstly, add $\log e_{i j} \in \mathrm{N}\left(0,2^{2}\right)$ to $\boldsymbol{A}_{0}$, we construct the inconsistent comparison matrix $\boldsymbol{A}_{\mathbf{2}}$, as shown below.

$$
\boldsymbol{A}_{2}=\left[\begin{array}{ccccc}
1.0000 & 2.0940 & 0.0707 & 0.3996 & 0.0772 \\
0.4775 & 1.0000 & 1.0955 & 1.0712 & 2.1457 \\
14.1398 & 0.9128 & 1.0000 & 2.4378 & 0.1630 \\
2.5020 & 0.9334 & 0.4102 & 1.0000 & 2.4328 \\
12.9505 & 0.4660 & \mathbf{6 . 1 3 2 6} & 0.4110 & 1.0000
\end{array}\right]
$$

From $A_{2}$, we have $\lambda_{\max }=8.040$ and $C I=0.760$.

Furthermore we evaluate the consistency of $\boldsymbol{A}_{\mathbf{2}}$ based on directed graph, by using our method stated in [Nishizawa, 1995].

Directed graph of $\boldsymbol{A}_{\mathbf{2}}$ is shown in Figure 1. There are some cycles in Figure 1, for example we have

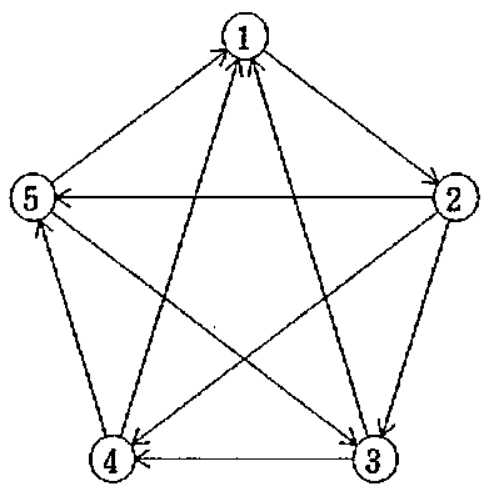

Figure 1. Directed graph of Example 2

cycle(1 2 3). By using our method stated in [Nishizawa,1997], we obtain 10 kind of various length of cycles. All cycles of Example 2 are shown in Table 2.

To improve consistency, we may eliminate the cycles. Therefore, we may find the arcs which do the minimum cover of the cycles. The cycle-arc incidence matrix is shown in Table 3 . For example, in Table 3 , cycle $(123)$ is consists of arcs $(1,2),(1,3)$, and $(2,3)$. In the cycle-arc incidence matrix 
Table 2. All cycles of Example 2

\begin{tabular}{|c|c|c|}
\hline length 3 & length 4 & length 5 \\
\hline$\left(\begin{array}{lll}1 & 2 & 3\end{array}\right)$ & $\left(\begin{array}{llll}1 & 2 & 3 & 4\end{array}\right)$ & $(12345)$ \\
\hline$\left(\begin{array}{lll}1 & 2 & 4\end{array}\right)$ & $\left(\begin{array}{llll}1 & 2 & 4 & 5\end{array}\right)$ & $(12453)$ \\
\hline 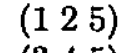 & 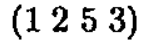 & 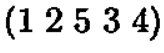 \\
\hline
\end{tabular}

Table 3. Cycle-arc incidence matrix for Example 2

\begin{tabular}{|c|c|c|c|c|c|c|c|c|c|c|}
\hline cycle/arc & $(1,2)$ & $(1,3)$ & $(1,4)$ & $(1, \overline{5})$ & $(2,3)$ & $(2,4)$ & $(2,5)$ & $(3,4)$ & $(3,5)$ & $(4,5)$ \\
\hline (123) & 1 & 1 & 0 & 0 & 1 & 0 & 0 & 0 & 0 & 0 \\
\hline$\left(\begin{array}{lll}1 & 2 & 4\end{array}\right)$ & 1 & 0 & 1 & 0 & 0 & 1 & 0 & 0 & 0 & 0 \\
\hline$(125)$ & 1 & 0 & 0 & 1 & 0 & 0 & 1 & 0 & 0 & 0 \\
\hline$(345)$ & 0 & 0 & 0 & 0 & 0 & 0 & 0 & 1 & 1 & 1 \\
\hline$(1234)$ & 1 & 0 , & 1 & 0 & 1 & 0 & 0 & 1 & 0 & 0 \\
\hline 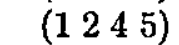 & 1 & 0 & 0 & 1 & 0 & 1 & 0 & 0 & 0 & 1 \\
\hline 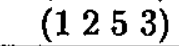 & 1 & 1 & 0 & 0 & 0 & 0 & 1 & 0 & 1 & 0 \\
\hline$(12345)$ & 1 & 0 & 0 & 1 & 1 & 0 & 0 & 1 & 0 & 1 \\
\hline$(12453)$ & 1 & 1 & 0 & 0 & 0 & 1 & 0 & 0 & 1 & 1 \\
\hline 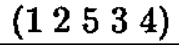 & 1 & 0 & 1 & 0 & 0 & 0 & 1 & 1 & 1 & 0 \\
\hline
\end{tabular}

these arcs be 1 and the other arcs be 0 . From Table 3 , the minimum covering arc is $(1,2)$. However it is not success to eliminate cycle (345).

To improve consistency of $\boldsymbol{A}_{2}$, based on directed graph, we can suggest that $a_{2_{12}}$ is incorrect. and $a_{234}, a_{245}$ and $a_{235}$ are need to a fresh comparison.

On the other hand by using proposed methos, similar procedure of Example 1, we have improved. comparison matrix $\boldsymbol{A}_{2}^{\prime}$ at twice iteration, as shown below.

$$
A_{2}^{\prime}=\left[\begin{array}{lllll}
1.0000 & 0.3280 & 0.2454 & 0.2874 & 0.1974 \\
3.0480 & 1.0000 & 0.7481 & 0.8760 & 0.6019 \\
4.0742 & 1.3366 & 1.0000 & 1.1709 & 0.8046 \\
3.4794 & 1.1415 & 0.8540 & 1.0000 & 0.6871 \\
5.0635 & 1.6612 & 1.2428 & 1.4552 & 1.0000
\end{array}\right]
$$

The end of improvement, we have $\lambda_{\max }=5$ and $C I=0$ and directed graph of $A_{2}^{\prime}$ is obtained in Figure 2. Compare Figure 1 with Figure 2, as a result, all the cycles, in Table 2, are eliminated.

Furthermore, as to compare Figure 2 with the perfect consistent case, directed graph of $\boldsymbol{A}_{\mathrm{o}}$ is shown in Figure 3. The only difference between Figure 2 and Figure 3 is a direction of $\operatorname{arc}(3,4)$.

Each weight of $A_{0}, A_{2}$ and $A_{2}^{\prime}$ and corresponding $R S S$ are shown in Table 4.

As a result, from Table 4, each weight of $\boldsymbol{A}_{2}^{\prime}$ is not entirely closer to the correct weight than each weight of $\boldsymbol{A}_{\mathbf{2}}$. However $\boldsymbol{A}_{2}^{\prime}$ is better than $A_{2}$ with respect to $R S S$.

\section{Conclusion}

In this paper we proposed a method to improve consistency of the comparison matrix in AHP. By applying our method to the artificially constructed inconsistent comparison matrices, as a result 


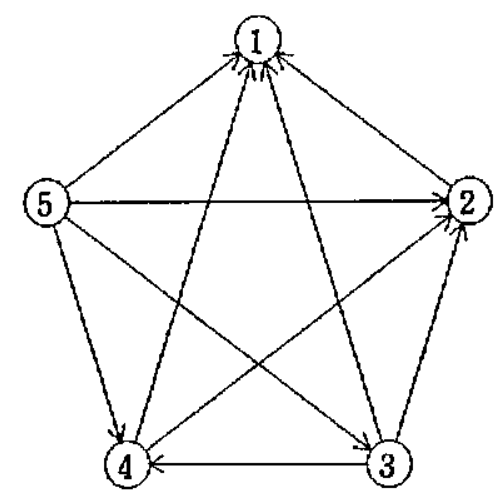

Figure 2. Directed graph of improved Example 2

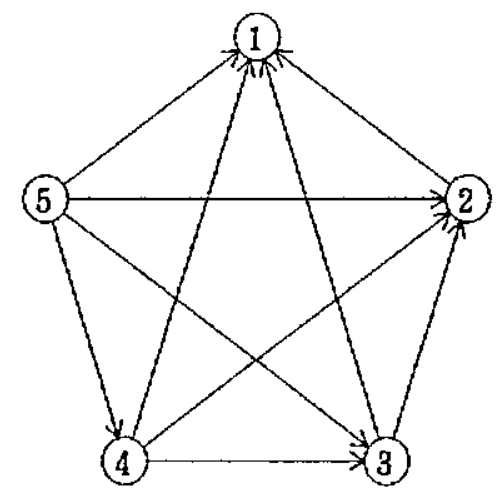

Figure 3. Directed graph of the perfect consistent case

Table 4. Each weight and corresponding $R S S$ of Example 2

\begin{tabular}{ccccccc}
\hline & $w_{1}$ & $w_{2}$ & $w_{3}$ & $w_{4}$ & $w_{5}$ & $R S S\left(\times 10^{-3}\right)$ \\
\hline $\boldsymbol{A}_{\circ}$ & .06666 & .13333 & .20000 & .26666 & .33333 & \\
$\boldsymbol{A}_{2}$ & .06807 & .17380 & .22988 & .18072 & .34752 & 10.1196 \\
$\boldsymbol{A}_{\mathbf{2}}^{\prime}$ & .06000 & .18290 & .24447 & .20878 & .30383 & 8.6994 \\
\hline
\end{tabular}


in each case, the values of $C I$ and $R S S$ are improved and in corresponding directed graph various length of the cycles are eliminated. Thus, we were able to confirm the effectiveness of the proposed method. Furthermore we need more discussion for highly inconsistent case and an incomplete information case.

\section{References}

[Saaty, 1977] Saaty, T. L. (1977) A Scaling Method for Priorities in Hierarchical Structures, Joumal of Mathematical Psychology, Vol. 15, 234-281.

[Saaty,1980] Saaty, T. L. (1980) The Analytic Hierarchy Process, McGrawHill, NewYork.

[Tone,1990] Tone, K (1990) Gēmu kankaku ishi-kettei-hou : Introduction to the AHP (in Japanese), Nikka-giren, 38-40.

[Nishizawa,1995] Nishizawa, K (1995) A Consistency Improving Method in Binary AHP, Journal of Operations Research Society of Japan, Vol. 38, No. 1, 21-33.

[Nishizawa,1997] Nishizawa, K (1997) A Method to Find Elements of Cycles in an Incomplete Directed Graph and Its Applications -Binary AHP and Petri Net -, An International Journal Computers $\mathcal{B}$ Mathematics with Applications, Vol.33, No.9,33-46. 